\title{
Paraaortic lymph node micrometastasis and tumor cell microinvolvement in advanced gastric carcinoma
}

\author{
Shoji Natsugoe, Saburo Nakashima, Masataka Matsumoto, Akihiro Nakajo, Futoshi Miyazono, \\ Fumio Kijima, Sumiya Ishigami, Kuniaki Aridome, Shuichi Hokita, Masamichi Baba, Sonshin Takao, \\ and TAKashi Aikou
}

First Department of Surgery, Kagoshima University School of Medicine, 8-35-1 Sakuragaoka, Kagoshima 890-8520, Japan

\begin{abstract}
:
Background. Paraaortic lymph node dissection in advanced gastric carcinoma is controversial. The purpose of this study was to investigate the incidence and significance of micrometastasis (MM) or tumor cell microinvolvement (TCM) in these critical lymph nodes.

Methods. A total of 2339 lymph nodes, including 390 paraaortic nodes, obtained from 47 patients with advanced gastric carcinoma were examined immunohistochemically, using cytokeratin antibody.

Results. Lymph node metastasis was found in 95 of the 390 paraaortic nodes of 14 patients by routine histological examination. MM or TCM was immunohistochemically detected in 45 of the 295 negative paraaortic lymph nodes from 15 of 33 patients $(\mathrm{MM}, n=5$; TCM, $n=10)$. The 5-year-survival rate in the paraaortic node-negative group and cytokeratinpositive group was significantly higher that that of the hematoxilin and eosin-positive group. The total number of lymph node metastases by hematoxylin and eosin staining and the pathological lymph node compartments, by cytokeratin-positive nodes, were prognostic factors by multivariate analysis.

Conclusions. We demonstrated a high rate of MM or TCM in the paraaortic lymph nodes and suggest that such harbored metastases are related to the prognosis of patients with advanced gastric carcinoma. On the basis of this study, a multi-institutional study should be considered.
\end{abstract}

Key words: advanced gastric cancer, lymph node metastasis, micrometastasis, immunohistochemistry, cytokeratin

\section{Introduction}

Lymph node metastasis is one of the most important factors affecting prognosis in patients with gastric cancer [1-4] and the appropriate extent of lymph

Offprint requests to: S. Natsugoe

Received for publication on June 7, 1999; accepted on Sept. 30, 1999 node dissection has been discussed in the literature. Some authors have reported that extended lymph node dissection contributes to improving the prognosis of node-negative patients [5], a finding which suggests the presence of occult lymph node metastasis that is not detectable by routine histopathologic examination [6,7]. Although the paraaortic lymph nodes are distant from the stomach, patients with advanced gastric carcinoma with paraaortic lymph node metastasis have been documented [8-10]. However, no unanimity currently exists in regard to the indications for and therapeutic effects of paraaortic lymphadenectomy.

The development of sensitive immunohistochemical techniques that rely on specific monoclonal antibodies has made possible the detection of small clusters of tumor cells, or even single tumor cells, in histologic tissue sections. Several groups have used cytokeratin expression as an epithelial marker for the specific detection of individual tumor cells and cell clusters in the lymph nodes of various types of carcinoma, including that of breast $[11,12]$, colon $[13,14]$, lung $[15,16]$, and stomach $[17,18]$. However, to our knowledge, no studies to date have examined tumor cell spread to the paraaortic lymph nodes in gastric carcinoma and the potential impact on patient prognosis.

Tumor cell spread to regional lymph nodes has not always shown a correlation to patient survival and the probability of recurrence [19], and it has been questioned whether, in these studies, the tumor cells detected in the regional lymph nodes of tumors truly represent metastases or whether they are an "epiphenomenon" which has very little, if any, impact on the patient's prognosis. A concept which may help to resolve this controversy is the idea that deposits of tumor cells found in lymph nodes may be classified as metastatic or non-metastatic. For this reason, we defined "micrometastasis" (MM) as tumor cells with a stromal reaction (granulation tissue or desmoplastic connective tissue) and "tumor cell microinvolvement" 
(TCM) as tumor cells without a stromal reaction $[17,20]$. The purpose of the present study was to clarify the incidence of MM or TCM in paraaortic lymph node metastasis in patients with advanced gastric carcinoma and to determine its clinical significance.

\section{Patients and methods}

\section{Study group}

Forty-seven patients (28 men and 19 women) with advanced gastric carcinoma were enrolled in this study between 1990 and 1995. All were preoperatively suspected of having abdominal lymph node metastasis by imaging techniques. They underwent gastrectomy with extended lymphadenectomy, including the paraaortic lymph nodes, at the First Department of Surgery, Kagoshima University Hospital, Japan. The areas of paraaortic lymph node removal, classified according to the Japanese Classification of Gastric Carcinoma [21], were: lymph nodes from the upper margin of the celiac trunk to the lower margin of the left renal vein (station no. 16a2), and lymph nodes from the lower margin of the left renal vein to the upper margin of the inferior mesenteric artery (station no. 16b1). However, the extent of paraaortic lymph node dissection was not specified (i.e., dissection of selected nodes or systematic dissection). None of the patients received radiation therapy or chemotherapy before surgical treatment and all were followed-up after discharge for a median follow-up period of 24 months (range, 1-94 months). Two patients died of postoperative complications (pneumonia and anastomotic leakage, respectively), and operative mortality was $4.3 \%$.

The ages of the patients ranged from 22 to 76 years (mean, 58.5 years). Based on the Japanese Classification of Gastric Carcinoma [21], 20 tumors were located in the upper third of the stomach, 6 in the middle third, 11 in the lower third, and 10 were distributed throughout the whole stomach. Histologically, the lesions were classified as differentiated adenocarcinoma, which included papillary and tubular tumors, or undifferentiated adenocarcinoma, which included poorly differentiated, signet-ring cell, and mucinous tumors. In total, 17 lesions were diagnosed as being differentiated adenocarcinoma, and 30 as undifferentiated adenocarcinoma. The depth of tumor invasion was $\mathrm{T} 1$ in 2 patients, $\mathrm{T} 2$ in 22, T3 in 17, and T4 in 6 (Table 1).

A total of 2399 lymph nodes were obtained from the 47 patients; the number of dissected nodes per patient ranged from 20 to 111 (median, 51.0). Of these nodes, 390 were paraaortic lymph nodes (average number of dissected paraaortic lymph nodes, 8.3; range, 2-35). All dissected lymph nodes were examined by conventional hematoxylin and eosin staining (H\&E) for metastasis
Table 1. Profile of the 47 patients with advanced gastric carcinoma

\begin{tabular}{lc}
\hline Features & $n$ \\
\hline Mean age (years) & $58.5 \pm 11.6$ \\
Sex ratio (M:F) & $28: 19$ \\
Tumor depth & 2 \\
T1 & 22 \\
T2 & 17 \\
T3 & 6 \\
T3 & \\
Histology & 17 \\
Tub & 25 \\
Por & 3 \\
Muc & 2 \\
Sig & \\
Lymph node metastasis & 9 \\
N0 & 16 \\
N1 & 5 \\
N2 & 17 \\
N3 & \\
\hline
\end{tabular}

Tub, Tubular adenocarcinoma; sig, signet ring cell carcinoma; por, poorly differentiated adenocarcinoma; N0, no lymph node metastasis; $\mathrm{N} 1$; metastasis to compartment 1 ; $\mathrm{N} 2$, metastasis to compartment 2; $\mathrm{N} 3$, metastasis to compartment 3

on one representative section cut at the plane with the largest diameter, including the hilus of the node.

\section{Immunohistochemistry}

All specimens were formalin-fixed and paraffinembedded. One additional section from each lymph node was stained immunohistochemcally, using AE1/ AE3 (20:1 mixture of AE1 to AE3; Boehringer, Mannheim, Germany), a monoclonal antibody cocktail which is reactive with a broad spectrum of human cytokeratins (CK) $[22,23]$. All sections were incubated at $60^{\circ} \mathrm{C}$ overnight. The tissue sections were then deparaffinized in xylene and rehydrated with a series of graded ethanols. After the slides were cooked in a pressure cooker in citrate buffer solution ( $\mathrm{pH}$ 6.0) for $6 \mathrm{~min}$, the sections were incubated with CK monoclonal antibody at a 1:100 dilution. The reactions for $\mathrm{CK}$ were developed with an alkaline phosphate anti-keratin phosphatase technique (APAAP method) [24]. The immunohistochemical study was limited to one slide per lymph node to simulate routine histological procedures. The negative control consisted of sections treated with the same protocol but with the primary antibody omitted. Normal gastric mucosa and the primary tumors of the specimens were used as positive controls and were consistently positive.

\section{Evaluation of results}

Tumor involvement of lymph nodes determined by immunohistochemistry was classified into two categories: 
(1) $\mathrm{MM}$ - tumor micrometastasis; cluster formation with a stromal reaction (granulation tissue or desmoplastic connective tissue) (Fig. 1) and (2) TCM - tumor microinvolvement; individual tumor cells without a stromal reaction (Fig. 2). The immunohistochemical results were independently examined by two observers (Sh.N. and Sa.N) without knowledge of the clinicopathologic data. Survival data were analyzed by the Kaplan-Meier survival model and expressed as observed overall survival, with data for two patients who died postoperatively being excluded. Statistical analysis was performed by the $\chi^{2}$ test and the Kruskal-Wallis test for group differences. The Kaplan-Meier analysis was also evaluated by the Log-rank test. Survival data were examined statistically in relation to the following set of covariables: sex; age; depth of tumor invasion; tumor size; histology; number of H\&E-positive nodes; number of CK-positive nodes; type of tumor cell

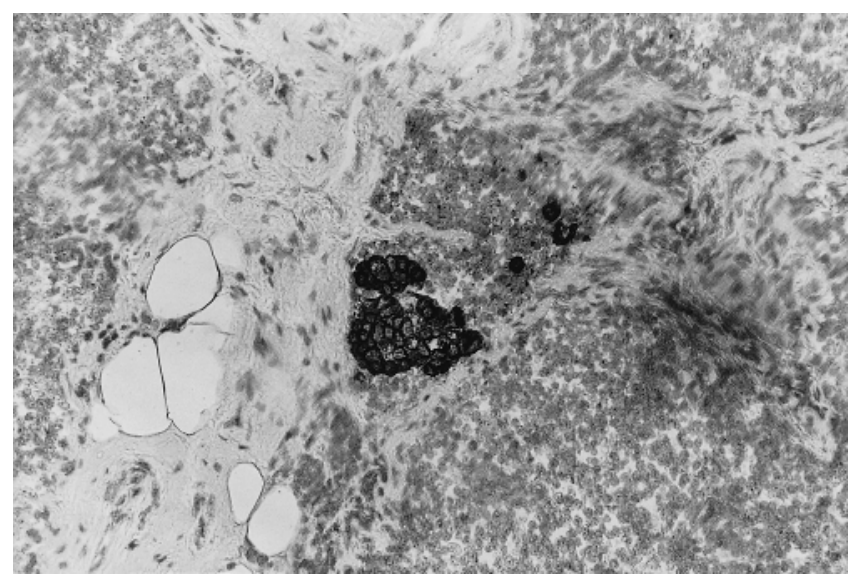

Fig. 1. Micrometastasis in lymph node, with cytokeratin antibody. $\times 200$

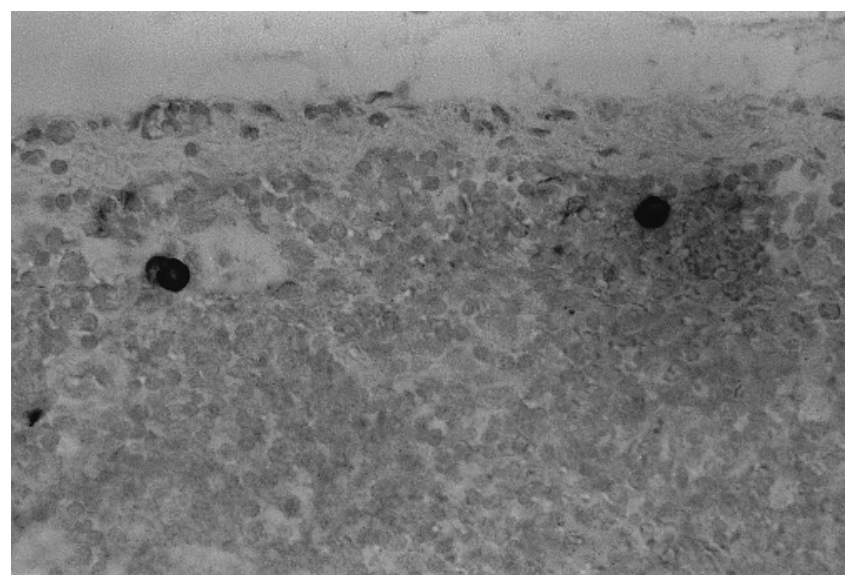

Fig. 2. Microinvolvement in lymph node, with cytokeratin antibody. $\times 400$ involvement (H\&E-positive, micrometastasis, tumor cell microinvolvement); and pathological lymph node compartment, by CK-positive nodes ( $\mathrm{pN} 0, \mathrm{pN} 1, \mathrm{pN} 2$, $\mathrm{pN} 3$ ). Prognostic factors were tested by univariate and multivariate analyses (proportional hazard regression model).

A $P$ value less than 0.05 was considered statistically significant.

\section{Results}

Lymph node metastasis was found in 38 of the 47 patients $(80.9 \%)$ by routine histological examination using H\&E. Of the 2399 nodes obtained by lymph node dissection, 630 nodes $(26.3 \%)$ had been involved by cancer cells. When the lymph nodes were examined immunohistochemically, tumor involvement in lymph nodes was found in 45 of the 47 patients $(95.7 \%)$ and in 875 of the 2399 nodes (36.5\%). Accordingly, tumor involvement was newly detected in 7 patients and 245 nodes. The number of patients with MM with or without TCM and with TCM alone, according to the depth of tumor invasion and histology, is shown in Table 2. Since the numbers in each category were small, a positive trend between the presence of MM and TCM and tumor depth or histology was not evident.

Initial routine histological examination revealed involved lymph nodes in 95 of the 390 paraaortic nodes $(24.4 \%)$ of 14 patients. Paraaortic lymph node metastases were newly detected by immunohistochemistry in $45(15.3 \%)$ of the 295 nodes negative for paraaortic lymph node metastasis by routine histological examination, in 15 of 33 patients ( $45.5 \%)$. The profiles of these 15 patients are shown in Table 3. Four patients had been diagnosed as being negative for all dissected lymph nodes. However, MM or TCM was found in compartment 1 and/or 2 lymph nodes. Therefore, all patients with MM or TCM in paraaortic nodes had other tumor involvement in the perigastric or in compartment 2 nodes. All patients had tumors with deeper than submucosal involvement. The total number of lymph nodes which showed tumor involvement was 89 in H\&E sections and 195 in CK sections. Tumor involvement in paraaortic nodes was newly detected in 26 nodes of 15 patients.

If MM and TCM are regarded as the initial forms of lymph node metastasis, then paraaortic node metastasis can be classified into three groups: negative, paraaortic lymph node metastasis not found either histologically or immunohistochemically; CK-positive, paraaortic lymph node metastasis detected only by immunohistological examination, and H\&E-positive, initial histological examination revealed paraaortic lymph node metastasis. The 5-year-survival rates for the negative, CK-positive, 
Table 2. Numbers of patients with micrometastasis and tumor cell microinvolvement according to depth of tumor invasion and histology

\begin{tabular}{lccc}
\hline & $\begin{array}{c}\text { Metastasis by H\&E } \\
(n=38)\end{array}$ & $\begin{array}{c}\text { MM } \pm \text { TCM } \\
(n=24)\end{array}$ & $\begin{array}{c}\text { TCM alone } \\
(n=21)\end{array}$ \\
\hline Tumor depth & & & 0 \\
T1 $(n=2)$ & 2 & 1 & 13 \\
T2 $(n=22)$ & 14 & 8 & 6 \\
T3 $(n=17)$ & 17 & 11 & 2 \\
T4 $(n=6)$ & 5 & 4 & 10 \\
Histology & 12 & 6 & 9 \\
Tub $(n=17)$ & 22 & 15 & 1 \\
Por $(n=25)$ & 2 & 2 & 1 \\
Muc $(n=3)$ & 2 & 1 & \\
Sig $(n=2)$ & & & \\
\hline
\end{tabular}

H\&E, Hematoxylin and eosin staining; MM, micrometastasis; TCM, tumor cell microinvolvement; tub, tubular adenocarcinoma; por, poorly differentiated adenocarcinoma; muc, mucinous adenocarcinoma; sig, signet-ring cell carcinoma

Table 3. Clinicopathological findings in the 15 patients with paraaortic lymph node micrometastasis or tumor cell microinvolvement

\begin{tabular}{|c|c|c|c|c|c|c|c|}
\hline $\begin{array}{l}\text { Patient } \\
\text { number }\end{array}$ & $\mathrm{n}$ & $\begin{array}{l}\text { Tumor } \\
\text { depth }\end{array}$ & Histology & $\begin{array}{c}\text { No. of LN meta } \\
\text { by H\&E }\end{array}$ & $\begin{array}{c}\text { No. of LN meta } \\
\text { by CK }\end{array}$ & $\begin{array}{l}\text { No. of paraaortic } \\
\text { LN meta by CK }\end{array}$ & $\begin{array}{c}\text { LN meta } \\
\text { pattern }\end{array}$ \\
\hline 1 & 0 & $\mathrm{~T} 2$ & Tub & 0 & 2 & 1 & MM \\
\hline 2 & 0 & $\mathrm{~T} 4$ & Sig & 0 & 5 & 2 & TCM \\
\hline 3 & 0 & $\mathrm{~T} 2$ & Tub & 0 & 6 & 3 & TCM \\
\hline 4 & 0 & $\mathrm{~T} 2$ & Tub & 0 & 10 & 3 & TCM \\
\hline 5 & 1 & $\mathrm{~T} 2$ & Por & 1 & 11 & 1 & TCM \\
\hline 6 & 1 & $\mathrm{~T} 2$ & Tub & 3 & 4 & 1 & MM \\
\hline 7 & 1 & $\mathrm{~T} 2$ & Tub & 3 & 11 & 2 & TCM \\
\hline 8 & 1 & T3 & Por & 3 & 13 & 1 & MM \\
\hline 9 & 1 & $\mathrm{~T} 3$ & Por & 5 & 25 & 2 & TCM \\
\hline 10 & 2 & T3 & Tub & 5 & 9 & 2 & TCM \\
\hline 11 & 2 & $\mathrm{~T} 4$ & Por & 7 & 12 & 1 & TCM \\
\hline 12 & 2 & $\mathrm{~T} 2$ & Por & 11 & 21 & 2 & TCM \\
\hline 13 & 2 & $\mathrm{~T} 3$ & Por & 14 & 17 & 2 & TCM \\
\hline 14 & 3 & $\mathrm{~T} 2$ & Tub & 10 & 15 & 2 & TCM \\
\hline 15 & 3 & $\mathrm{~T} 3$ & Por & 27 & 34 & 1 & MM \\
\hline Total & & & & 89 & 195 & 26 & \\
\hline
\end{tabular}

n, Lymph node compartment; LN meta, lymph node metastasis; H\&E, hematoxylin and eosin staining; CK, cytokeratin staining; tub, tubular adenocarcinoma; sig, signet ring cell carcinoma; por, poorly differentiated adenocarcinoma; MM, micrometastasis; TCM, tumor cell microinvolvement

and H\&E-positive groups were $56.0 \%, 25.2 \%$, and $9.0 \%$, respectively (Fig. 3). The survivals in the negative group and CK-positive group were significantly higher than that of the H\&E-positive group $(P<0.001$ and $P=$ 0.03 , respectively). In the CK-positive group, the survival time of patients with MM was significantly shorter than that of patients with TCM $(P=0.04)$ (Fig. 4).

Factors related to prognosis were evaluated by univariate and multivariate analyses. According to the univariate analysis, tumor size, number of H\&Epositive nodes, number of CK-positive nodes, and pathological lymph node compartment (by CK-positive nodes; $\mathrm{pN} 0, \mathrm{pN} 1, \mathrm{pN} 2, \mathrm{pN} 3)$ were related to prognosis. According to the multivariate regression analysis, the number of H\&E-positive nodes and pathological lymph node compartment, by CK-positive nodes, were independent prognostic factors (Table 4).

\section{Discussion}

Improved imaging techniques have enabled lymph node metastasis to be more accurately demonstrated preoperatively. However, the detection of small metastatic 

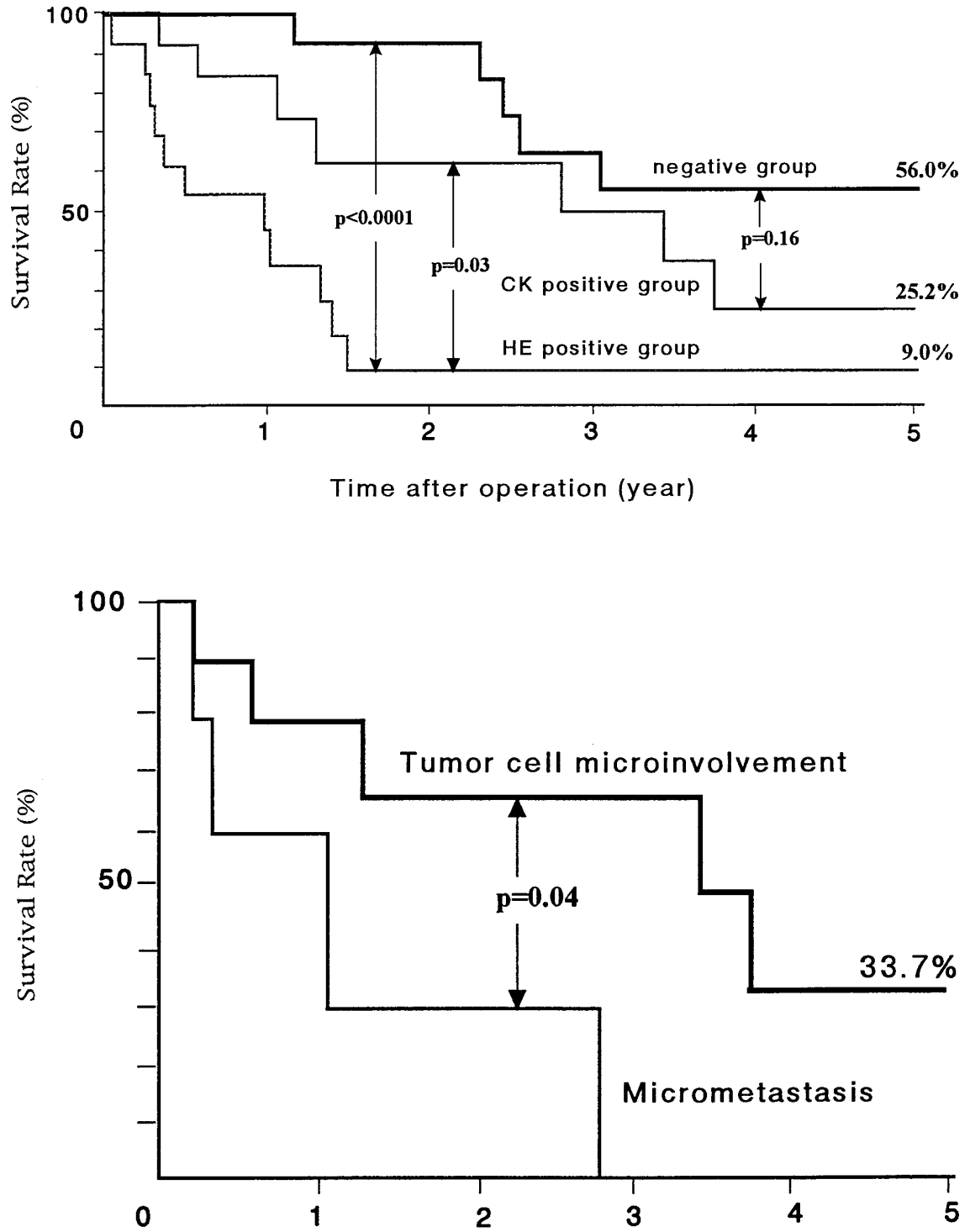

Time after operation (year)
Fig. 3. Five-year-survival rates according to lymph node metastasis; negative group, cytokeratin $(C K)$-positive group, and hematoxilin and eosin $(H E)$-positive group. See text for explanation of groups

Fig. 4. Five-year-survival rates according to metastatic pattern in paraaortic lymph nodes

Table 4. Risk factors affecting overall survival rate determined by univariate and multivariate analyses of prognostic factors

\begin{tabular}{|c|c|c|c|c|}
\hline Independent factors & $\begin{array}{c}\text { Univariate } \\
\text { ( } P \text { value })\end{array}$ & $\begin{array}{c}\text { Multivariate } \\
\text { ( } P \text { value })\end{array}$ & Relative risk & $95 \% \mathrm{CI}$ \\
\hline No. of H\&E-positive nodes & 0.0004 & 0.0165 & 1.017 & $1.033-1.359$ \\
\hline pN, by CK-positive nodes & 0.0045 & 0.0245 & 1.697 & $1.071-2.690$ \\
\hline
\end{tabular}

CI, Confidence interval; H\&E, hematoxylin and eosin staining; $\mathrm{pN}$, pathological lymph node compartment; $\mathrm{CK}$, cytokeratin staining

foci in the lymph nodes is still very difficult. In some studies, micrometastasis has been identified by detailed histological examination in additional sections [25,26], and a high incidence of micrometastases has recently been detected in gastric cancer by immunohistochemistry $[17,18]$. The present study focused on paraaortic lymph node metastasis because the paraaortic lymph nodes are thought to be critical in terms of decision- 
making for extended lymph node dissection in gastric cancer surgery. Recent developments in perioperative management have enabled extended lymph node dissection that includes the paraaortic lymph nodes to be safely performed in patients with gastric cancer $[8,9,27]$. For planning any kind of adjuvant treatment, it would be useful to examine for MM or TCM in paraaortic nodes to identify patients with poorer prognosis than those without MM or TCM.

In the present study, we found a high incidence of micrometastasis in the paraaortic nodes of patients with advanced gastric carcinoma. The total number of metastatic nodes in the 15 patients in whom paraaortic lymph node metastasis was newly found ranged from 1 to 20. These patients had involvement in other nodes as well as in the paraaortic nodes. Furthermore, paraaortic lymph node metastases were detected immunohistochemically in 4 patients diagnosed as having negative nodes by routine histological examination. In these patients, MM and/or TCM were also found in the lymph nodes of compartment 1 and/or 2. This result suggested that paraaortic lymph node metastasis occurred via nodes at other sites, for example, via the perigastric nodes and nodes along the left gastric artery.

The 5-year survival in the 15 patients was best in the negative group, followed by the CK-positive group and the H\&E-positive group in descending order. Furthermore, patients with TCM in the paraaortic lymph nodes had a significantly better prognosis than those with MM. From the analysis of prognosis, some patients benefited by paraaortic lymphadenectomy and others did not. According to our multivariate regression analysis, the number of H\&E-positive nodes and the pathological lymph node compartment, by CK-positive nodes, were independent prognostic factors. This result suggests that examining for the presence of MM and TCM would be clinically, useful, as immunohistochemical CK staining in the lymph nodes was one of the prognostic factors. However, as the number of patients, in this study was small, a future multiinstitutional study will be necessary to confirm these findings.

In conclusion, we detected a high incidence of MM and TCM in the paraaortic lymph nodes in patients with advanced gastric carcinoma. This finding indicates that immunohistochemical staining for $\mathrm{CK}$ in the lymph nodes, including paraaortic lymph nodes, in patients with gastric carcinoma is clinically useful to evaluate the prognosis of patients and to plan postoperative adjuvant treatment.

Acknowledgments This study was supported in part by Grants-in-Aid for Scientific Research from the Ministry of Education, Science and Culture, Japan.

\section{References}

1. Maruyama K, Gunvén P, Okabayashi K, Sasako M, Kinoshita T. Lymph node metastases of gastric cancer. Ann Surg 1989;210: 596-602.

2. Siewert JR, Böttcher K, Roder JD, Busch R, Hermanek P, Meyer HJ, German Gastric Carcinoma Study Group. Prognostic relevance of systemic lymph node dissection in gastric carcinoma. $\mathrm{Br}$ J Surg 1993;80:1015-8.

3. Manzoni GD, Verlato G, Guglielmi A, Laterza E, Genna M, Cordiano C. Prognostic significance of lymph node dissection in gastric cancer. Br J Surg 1996;83:1604-7.

4. Kodera Y, Yamamura Y, Shimizu Y, Torii A, Hirai T, Yasui K, et al. Metastatic gastric lymph node rate is a significant prognostic factor for resectable stage IV stomach cancer. J Am Coll Surg 1997;185:65-9.

5. Maehara Y, Tomoda M, Tomisaki S, Ohmori M, Baba H, Akazawa K, et al. Surgical treatment and outcome for nodenegative gastric cancer. Surgery 1997;121:633-9.

6. Gunvén P, Maruyama K, Okabayashi K, Sasako M, Kinoshita T. Non-ominous micrometastases of gastric cancer. $\mathrm{Br} \mathrm{J}$ Surg 1991;78:352-4.

7. Maehara Y, Okuyama T, Moriguchi S, Orita H, Kusumoto H, Korenaga D, et al. Prophylactic lymph node dissection in patients with advanced gastric cancer promotes increased survival time. Cancer 1992;70:392-5.

8. Kitamura M, Arai K, Iwasaki Y. Clinical studies on surgical benefit of paraaortic lymph node dissection in gastric cancer patients from the point of quality of life and outcome. Gekachiryo (Surgical Therapy) 1995;73:301-6.

9. Isozaki H, Okajima K, Fujii K, Nomura E, Izumi N, Ohyama T, et al. Significance and indication of super-extended lymph node dissection (D4) for gastric cancer. Shokakigeka (Gastroenterological Surgery) 1997;20:539-50.

10. Aikou T, Natsugoe S, Hokita S. Extended gastric surgery: is paraaortic lymph node dissection essential for advanced gastric cancer? Gan to Kagakuryoho (Jpn J Cancer Cehmother) 1998;25:498-503.

11. Galea MH, Athanassiou E, Bell J, Dilks B, Robertson J, Elston $\mathrm{CW}$, et al. Occult regional lymph node metastases from breast carcinoma: immunohistological detection with antibodies CAM 5.2 and NCRC-11. J Pathol 1991;165:221-7.

12. Nasser IA, Lee A, Bosari S, Saganich R, Heatley G, Silverman ML. Occult axillary lymph node metastases in "node-negative" breast carcinoma. Hum Pathol 1993;24:950-7.

13. Greenson JK, Isenhart CE, Rice R, Mojzisik C, Houchens D, Martin EW. Identification of occult micrometastases in pericolic lymph nodes of Dukes' B colorectal cancer patients using monoclonal antibodies against cytokeratin and CC49: correlation with long-term survival. Cancer 1994;73:563-9.

14. Jeffers MD, O'Dowd GM, Mulcahy H, Stagg M, O'Donoghue DP,Toner $\mathrm{M}$. The prognostic significance of immunohistochemically detected lymph node micrometastases in colorectal carcinoma. J Pathol 1994;172:183-7.

15. Chen ZL, Perez S, Holmes EC, Wang HJ, Coulson WF, Wen D, et al. Frequency and distribution of occult micrometastases in lymph nodes of patients with non-small-cell lung carcinoma. J Natl Cancer Inst 1993;85:493-8.

16. Passlick B, Izbicki JR, Kubuschok B, Nathrath W, Thetter O, Pichlmeier U, et al. Immunohistochemical assessment of individual tumor cells in lymph nodes with non-small-cell lung cancer. J Clin Oncol 1994;12:1827-32.

17. Siewert JR, Kestlmeier R, Busch R, Böttcher K, Roder JD, Meuller J, et al. Benefits of D2 lymph node dissection for patients with gastric cancer and $\mathrm{pN} 0$ and $\mathrm{pN} 1$ lymph node metastases. $\mathrm{Br}$ J Surg 1996;83:1144-7.

18. Ishida K, Katsuyama T, Sugiyama A, Kawasaki S. Immunohistochemical evaluation of lymph node micrometaastases from gastric carcinomas. Cancer 1997;79:1069-76. 
19. Friedman S, Bertin F, Mouriesse H, Benchabat A, Genin J, Sarrazin D, et al. Importance of tumor cells in axillary node sinus margins ("clandestine" metastases) discovered by serial sectioning in operable breast carcinoma. Acta Oncol 1988;27:4837.

20. Natsugoe S, Mueller J, Stein HJ, Feith M, Höfler H, Siewert JR. Micrometastasis and tumor cell microinvolvement of lymph nodes from esophageal squamous cell carcinoma: frequency, associated tumor characteristics, and impact on prognosis. Cancer 1998;83:858-66.

21. Japanese Gastric Cancer Association. Japanese classification of gastric carcinoma. 2nd English ed. Gastric Cancer 1998;1:115.

22. Woodcock MJ, Eichner R, Nelson WG, Sun TT. Immunolocalization of keratin polypeptides in human epidermis using monoclonal antibodies. J Cell Biol 1982;95:5808.
23. Listrom MB, Dalton LW. Comparison of keratin monoclonal antibodies MAK-6, AE1:AE3, and CAM-5.2. Am J Clin Pathol 1987;88:297-301.

24. Cordell JL, Falini B, Erber WN, Ghosh AK, Abdulaziz Z, Macdonald S, et al. Immunoenzymatic labeling of monoclonal antibodies using immune complexes of alkaline phosphatase and monoclonal anti-alkaline phosphatase (APAAP complexes). J Histochem Cytochem 1984;32:219-29.

25. International (Ludwig) Breast Cancer Study Group. Prognostic importance of occult axillary lymph node micrometastases from breast cancer. Lancet 1990;335:1565-8.

26. Natsugoe S, Aikou T, Shimazu H. A detailed histological study on occult metastasis of the lymph nodes. Jpn J Surg 1991;21:528-32.

27. Volpe CM, Koo J, Miloro SM, Driscoll DL, Nava HR, Douglass HO. The effect of extended lymphadenectomy on survival in patients with gastric adenocarcinoma. J Am Coll Surg 1995;181: 56-64. 